WIS/38/02-AUG-DPP

\title{
Null orbifolds in AdS, Time Dependence and Holography
}

\author{
Joan Simón \\ The Weizmann Institute of Science, Department of Particle Physics \\ Herzl Street 2, 76100 Rehovot, Israel \\ E-mail: jsimon@weizmann.ac.il
}

\begin{abstract}
We study M/D-branes in a null-brane background. By taking a near horizon limit, one is left with cosmological models in the corresponding Poincaré patches. To deal with their usual horizons, we either extend these models to global AdS or remain in the Poincaré patch and apply a T-duality transformation whenever the effective radius of the compact dimension associated with the null-brane probes distances smaller than the string scale. The first scenario gives rise to null orbifolds in AdS spaces, which are described in detail. Their conformal boundaries are singular. The second has a dual gauge theory description in terms of Super Yang-Mills in the null-brane background. The latter is a good candidate for a non-perturbative definition of string theory in a time-dependent background.
\end{abstract}

Keywords: null orbifolds, AdS spaces, AdS/CFT correspondence, time dependence 


\section{Introduction}

One of the most interesting questions in theoretical high energy physics concerns the lessons that a quantum theory of gravity might teach us about the origin of the universe. It is thus natural to study time dependence in the framework of string theory. Lately, there has been certain amount of work in this direction. One can consider orbifolds and discrete quotients of Minkowski spacetime acting non-trivially on time [1, 2, 3, 4, 5, 6, 7, 10, 8, 9, 11, 12]. Out of the different possibilities, there is a single basic one preserving one half of the spacetime supersymmetries, the null (parabolic) orbifold [1] acting on $\mathbb{R}^{1,2}$. This is a singular spacetime : it has fixed points, it is nonHausdorff and it has closed lightlike curves. These features are resolved by the null-brane [4, 8, 11, 12] which is obtained by adding a shift, transverse to $\mathbb{R}^{1,2}$, to the discrete quotient action. The stability of these configurations 13, 12, 14 and the validity of perturbative techniques have also been partially discussed [9, 11, 12, 14. Another possibility is that of coset models having a cosmological spacetime interpretation and allowing a conformal field theory description on the worldsheet [15, 16]. There are other approaches, such as considering certain double Wick rotations of previously known configurations [17, 18, 19, 20, 21, 22], S-branes [23, 24, 25, 26] and Sen's proposal 27, 28, 29 that dynamical rolling of the tachyon of open string field theory can lead to interesting cosmologies.

In this work, we shall be concerned with the first mentioned approach. The analysis made so far seems to indicate that the null-brane background is stable to black hole formation when probed by particles for large shifts and when the number of non-compact dimensions is big enough $(D>5)$ [12, 14]. However, our understanding of string dynamics in these backgrounds is still poor, specially concerning the winding sector. It is instructive to consider the open string sector by adding D-branes, expecting to get some insight from their low energy gauge theory description at weak coupling.

Our main goal will be to start exploring a possible non-perturbative string cosmology scenario by analysing the null-brane background in the context of the AdS/CFT correspondence [30, 31]. It is thus necessary to know which $\mathrm{M} / \mathrm{D}$-branes exist in this vacuum, the amount of supersymmetry they preserve and their low energy closed string description in terms of classical supergravity configurations. Most of these steps have been answered in [32, 33], or consist of generalisations of the techniques developed there. We shall primarily be concerned with branes in which the discrete quotient takes place along their worldvolumes. Even though the dynamics of string theory in these time dependent backgrounds is not understood, it is natural to study the near horizon geometry of the corresponding M2/M5/D3 configurations. 
The null-brane carries an intrinsic scale, that we shall denote by $R$, which gives the size of the neck of spacetime when moving from its contracting phase to its expanding one. It is natural to keep it fixed when taking the near horizon limit, which for D3-branes will be

$$
\alpha^{\prime} \rightarrow 0 \quad, \quad \mathrm{u}=\frac{\mathrm{r}}{\alpha^{\prime}}, \mathrm{g}_{s}, \mathrm{R} \quad \text { fixed }
$$

The resulting geometry gives rise to a cosmological model in the Poincaré patch of $\mathrm{AdS}_{5}$. The latter has a similar geometrical interpretation to the one in flat spacetime, but D3-branes, or more generally, any M/D-brane that allows such a construction gives rise to horizons at $\mathrm{u}=0$. There are two main roads that one can follow to understand the meaning of these horizons :

(i) Extend the cosmological model by crossing the horizon. This leads naturally to the analysis of orbifolds and discrete quotients of global AdS spaces.

(ii) Since there is a compact dimension along the brane, whenever its effective radius probes distances smaller than the string scale, one is allowed to apply T-duality and use its T-dual description to understand the original horizon.

The first approach leads naturally to consider the analysis of null orbifolds in AdS spaces 円. This is because Lorentz transformations in the Poincaré patch are mapped into Lorentz transformations in $\mathbb{R}^{2, p-1}$, the space in which we embed global $\mathrm{AdS}_{p}$. On the other hand, translations in the first turned out to be null rotations (boost + rotation) in $\mathbb{R}^{2, p-1}$, involving the "second" time. To study the geometry, structure of singularities and conformal boundary of these discrete quotients, the embedding of $\mathrm{AdS}_{p}$ in $\mathbb{R}^{2, p-1}$ turns out to be very useful. As expected, the null orbifold of AdS spaces has fixed points and it is a non-Hausdorff space, as it happened in the flat spacetime orbifold. Somewhat more surprisingly, the analogue of the null-brane discrete quotient in global $\mathrm{AdS}_{p}$ spaces $(p \geq 5)$ has no fixed points, but its conformal boundary is singular. It has fixed points, it is non-Hausdorff and it has closed lightlike curves. It can be shown that this singular conformal boundary is equivalent to the conformal compactification of the null-brane manifold. These statements may lead us to conclude that if there is any field theory dual to such cosmological scenarios in AdS, living on its boundary, its base space must be a singular manifold.

\footnotetext{
${ }^{1}$ There have been previous works on orbifolds and discrete quotients of AdS spaces in the context of AdS/CFT, see [34, 35, 36, 37].
} 
The second approach is much more appealing. Borrowing some intuition from the AdS/CFT correspondence, the cosmological model on the Poincaré patch of $\mathrm{AdS}_{5}$ should be dual to a four dimensional Super Yang-Mills (SYM) gauge theory in the null-brane background. Thus, a priori, this scenario has some perturbative description on the worldsheet, by extending the formalism developed in [9] for the null orbifold to the open string sector of the nullbrane discrete quotient. It has a known low energy effective supergravity description and a dual gauge theory. Furthermore, T-duality can be studied both on the worldsheet and on supergravity. All in all, these might be the necessary ingredients to provide a non-perturbative definition of string cosmology. We shall comment on the first steps to fulfill this programme.

The organisation of the paper is as follows. In section 2, we explain the main tools necessary to describe null orbifolds in $\operatorname{AdS}_{p}$ spaces, $\forall p \geq$ 3. As an aside remark, we briefly comment on the embedding of the BTZ black holes [38, 39] in higher dimensional AdS spaces. In section 3, we embed the previous discussion in string/M-theory, by extending our analysis to include the q-forms relevant to $A d S_{4} \times S^{7}, A d S_{7} \times S^{4}$ and $A d S_{5} \times S^{5}$ spacetimes and by discussing supersymmetry considerations. In section 4 , we comment on the corresponding orbifold construction in brane configurations and their near horizon limits. In particular, we analyse the mapping of symmetries between the Poincaré patch and global AdS. In section 5, we discuss the analogue of the null-brane discrete quotient in $\operatorname{AdS}_{p}$ spaces $(p \geq$ 5 ) and discuss the singularities of their conformal boundary. In section 6 , we comment on the gauge theory dual to the cosmological models in the Poincaré patches, some T-duality properties of our bulk configurations and on what we are still missing to fulfill this programme. Some explicit computations to prove the amount of supersymmetry preserved by the discrete quotients discussed in the body of the paper are performed in an appendix.

Note ADDED. While working on this project, we received [40] which discusses similar cosmological scenarios and their holographic duals in terms of time-dependent non-commutative field theories. Notice that in their scenario, the shift that gives rise to the null-brane is transverse to the brane (D2-brane in the forementioned reference), and so it describes a physically inequivalent model to the ones considered in this work.

\section{Null orbifolds in AdS spaces}

The p-dimensional anti-de Sitter space $\left(A d S_{p}\right)$ is a maximally symmetric space of constant negative curvature. It can be represented as the hyper- 
boloid

$$
\begin{gathered}
\operatorname{AdS}_{p} \hookrightarrow \mathbb{R}^{2, p-1} \\
-u^{2}-v^{2}+\sum_{i=1}^{p-1}\left(x^{i}\right)^{2}=-\left(\mathrm{R}_{\mathrm{AdS}}\right)^{2}
\end{gathered}
$$

in the flat $(\mathrm{p}+1)$-dimensional space $\mathbb{R}^{2, p-1}$. By construction, the space has the isometry group $\mathrm{O}(2, p-1)$ ?.

Equation (1) can be solved by setting

$$
\begin{aligned}
u & =\mathrm{R}_{\mathrm{AdS}} \cosh \rho \cos \tau \quad, \quad v=\mathrm{R}_{\mathrm{AdS}} \cosh \rho \sin \tau \\
x^{i} & =\mathrm{R}_{\mathrm{AdS}} \sinh \rho \Omega^{i} \quad\left(i=1, \ldots, p-1 ; \quad \sum_{i}\left(\Omega^{i}\right)^{2}=1\right),
\end{aligned}
$$

where $\left\{\Omega^{i}\right\}$ parametrise a unit (p-2)-sphere. The induced metric on the hyperboloid turns out to be

$$
g_{A d S_{p}}=\left(\mathrm{R}_{\mathrm{AdS}}\right)^{2}\left[-(\cosh \rho)^{2}(d \tau)^{2}+(d \rho)^{2}+(\sinh \rho)^{2} g_{S^{p-2}}\right]
$$

where $g_{S^{p-2}}$ stands for the metric on a unit $\mathrm{S}^{p-2}$ sphere. As usual in the physics literature, we shall refer to $\mathrm{AdS}_{p}$ to the universal covering space of the above hyperboloid (何) in which the global timelike coordinate $\tau$ has been unwrapped (i.e. take $-\infty<\tau<\infty$ ).

Any Killing vector $\xi$ defines a one parameter subgroup of isometries whose action in $\mathrm{AdS}_{p}$ is given by

$$
\mathrm{P} \rightarrow e^{t \xi} \mathrm{P} \quad \forall \mathrm{P} \in \mathrm{AdS}_{p}
$$

In this section, we shall be concerned with the subgroup generated by

$$
\xi^{ \pm}=\frac{1}{\sqrt{2}}\left[u \partial_{x}+x \partial_{u} \pm\left(y \partial_{x}-x \partial_{y}\right)\right]=x^{ \pm} \partial_{x}+x \partial_{\mp},
$$

where $x^{ \pm}=(u \pm y) / \sqrt{2}$. Notice that $\xi^{ \pm}$is the linear combination of a boost in the ux-plane and a rotation in the yx-plane. The quotient manifold $\operatorname{AdS}_{p} / \Gamma^{ \pm}$ is obtained by identifying points under the action (4) whenever $t$ is an integer multiple of a basic discrete step.

In the following, we shall discuss the geometry of these quotient manifolds both for global $A d S_{p}$, and for particular patches of it, the second option giving rise to the embedding of the BTZ black hole [38, 39] in higher dimensional $A d S_{p}$ spaces $(p \geq 4)$.

\footnotetext{
${ }^{2}$ When embedding these spaces in string theory, the presence of fluxes will restrict this isometry group to $\mathrm{SO}(2, p-1)$.

${ }^{3}$ The existence of such a generator requires us to work with $\operatorname{AdS}_{p} p \geq 3$.
} 


\subsection{Global AdS}

The embedding $A d S_{p} \hookrightarrow \mathbb{R}^{2, p-1}$ provides us with the most natural way to study the null orbifold $A d S_{p} / \Gamma^{+}$. Introduce coordinates $\left\{x^{+}, x^{-}, x\right\}$ on $\mathbb{R}^{1,2} \subset \mathbb{R}^{2, p-1}(p \geq 3)$, being defined as above and assembled them into a column vector $X$. The generator $g_{0}$ of the orbifold acts as

$$
X=\left(\begin{array}{c}
x^{+} \\
x \\
x^{-}
\end{array}\right) \rightarrow g_{o} \cdot X=e^{t \mathcal{J}} X=\left(\begin{array}{c}
x^{+} \\
x+t x^{+} \\
x^{-}+t x+\frac{1}{2} t^{2} x^{+}
\end{array}\right) ; \mathcal{J}=\left(\begin{array}{lll}
0 & 0 & 0 \\
1 & 0 & 0 \\
0 & 1 & 0
\end{array}\right)
$$

The above definition coincides with $\mathbb{R}^{1,2} / \Gamma^{+}$, but it certainly applies to $A d S_{p} / \Gamma^{+}$since the action of $\Gamma^{+}$leaves (1) invariant. Following the discussion in 8 , 9. the action of $\Gamma^{+}$on the embedding space $\mathbb{R}^{2, p-1}$ has a subset of fixed points located at $x^{+}=x=0$. Furthermore, $\mathbb{R}^{2, p-1} / \Gamma^{+}$is a non-Hausdorff space, as can be seen by inspection of the $x^{+}=0$ subspace. To learn about $A d S_{p} / \Gamma^{+}$, one evaluates (1) in these singular subspaces. Denoting $x^{p-2}=x$ and $x^{p-1}=y$, the set of fixed points in $A d S_{p} / \Gamma^{+}$is described by

$$
v_{ \pm}= \pm \sqrt{\left(\mathrm{R}_{\mathrm{AdS}}\right)^{2}+\sum_{i=1}^{p-3}\left(x^{i}\right)^{2}}
$$

consisting of two disconnected branches, whereas the loci where $\left\|\xi^{+}\right\|^{2}=$ $\left(x^{+}\right)^{2}=0$ is given by

$$
v_{ \pm}= \pm \sqrt{\left(\mathrm{R}_{\mathrm{AdS}}\right)^{2}+\sum_{i=1}^{p-3}\left(x^{i}\right)^{2}+(x)^{2}}
$$

The fact that $\left\{x^{+}=0\right\} \in \operatorname{AdS}_{p} \forall \mathrm{x}$ allows us to conclude that $A d S_{p} / \Gamma^{+}$is also a non-Hausdorff space.

Working in global coordinates (2) and picking $\Omega^{x}=\cos \theta$ and $\Omega^{y}=$ $\sin \theta \cos \psi \quad 0 \leq \theta, \psi<\pi$, for concreteness, we can describe the set of fixed points by

$$
\begin{aligned}
& \rho=0 \quad, \quad \tau=\frac{\pi}{2}+\pi \mathrm{n} \quad \mathrm{n} \in \mathbb{Z} \quad \forall \vec{\Omega} \in \mathrm{S}^{p-2} \\
& \theta=\frac{\pi}{2} \quad, \quad \cos \tau=-\tanh \rho \cos \psi \quad \forall \vec{\Omega} \in \mathrm{S}_{\sin \psi}^{p-4}
\end{aligned}
$$

where $\mathrm{S}_{\sin \psi}^{p-4}$ stands for a (p-4)-sphere of radius $\sin \psi$. 
It is particularly interesting to study the action of $\Gamma^{+}$on the conformal boundary of $\mathrm{AdS}_{p}$. It is well-known that $\mathrm{AdS}_{p}$ can be conformally mapped to one half of the Einstein static universe. This can be seen by introducing a new coordinate $\varphi$ related to $\rho$ by

$$
\tan \varphi=\sinh \rho \quad\left(0 \leq \varphi<\frac{\pi}{2}\right) .
$$

The $\mathrm{AdS}_{p}$ metric is conformal to

$$
g_{E(p)}=-(d \tau)^{2}+(d \varphi)^{2}+(\sin \varphi)^{2} g_{S^{p-2}} .
$$

It is now manifest that the spacelike hypersurfaces of constant $\tau$ are (p-1)hemispheres, whose equator $(\varphi=\pi / 2)$ is a boundary with topology $\mathrm{S}^{p-2}$. The boundary of the full $\operatorname{AdS}_{p}$ spacetime is thus $\mathbb{R} \times \mathrm{S}^{p-2}$, and located at $\varphi=\pi / 2(\rho \rightarrow \infty)$.

Moving to $\operatorname{AdS}_{p} / \Gamma^{+}$, one can derive the set of fixed points on the boundary by evaluating (7) at $\rho \rightarrow \infty$, giving rise to :

$$
\theta=\frac{\pi}{2} \quad, \quad \tau=\pi \pm \psi(\bmod 2 \pi) \quad \forall \vec{\Omega} \in \mathrm{S}_{\sin \psi}^{p-4}
$$

Using similar arguments as before, one concludes that the boundary is nonHausdorff due to the specific features of the hypersurface

$$
\cos \tau=-\sin \theta \cos \psi .
$$

The above analysis suggests that if there is any field theory dual living on the boundary, it must be defined on a singular base space $\mathcal{M}$, which we shall later identify with the conformal compactification of the null-brane manifold. Before discussing this point, we shall first find a useful local description for the orbifold $\operatorname{AdS}_{p} / \Gamma^{+}$. Inspired by the embedding $A d S_{p} \hookrightarrow \mathbb{R}^{2, p-1}$, it is natural to introduce a local adapted coordinate system $\left\{z^{+}, z, z^{-}\right\}$

$$
\begin{aligned}
x^{+} & =z^{+} \\
x & =z z^{+} \\
x^{-} & =z^{-}+\frac{1}{2} z^{+} z^{2}
\end{aligned}
$$

in which $\xi^{+}=\partial_{z}$, so that the identifications become shifts in the $z$ variable

$$
\left(z^{+}, z, z^{-}\right) \sim\left(z^{+}, z+2 \pi, z^{-}\right)
$$


in some convenient normalization. It is important to keep in mind that the coordinate system $\left\{z^{+}, z, z^{-}\right\}$defined in (9) breaks down at $z^{+}=0$. Rewriting (II) in terms of $\left\{z^{+}, z, z^{-}\right\}$

$$
-2 z^{+} z^{-}-v^{2}+\sum_{i=1}^{p-3}\left(x^{i}\right)^{2}=-\left(\mathrm{R}_{\mathrm{AdS}}\right)^{2},
$$

one discovers that it is independent of the compact variable $z$. One thus learns that for $z^{+} \neq 0$, the metric for the null orbifold $\mathrm{AdS}_{p} / \Gamma^{+}$is that of

$$
g_{A d S_{p} / \Gamma^{+}}=g_{A d S_{p-1}}+\left(z^{+}\right)^{2}(d z)^{2},
$$

that is, some $S^{1}$ fibration over $A d S_{p-1}$. Notice that $\operatorname{AdS}_{p-1}$ has the same radius $\mathrm{R}_{\mathrm{AdS}}$ as the original $\mathrm{AdS}_{p}$ and that the effective radius $\left(z^{+}\right)$of the compact direction ( $\mathrm{z}$ ) belongs to $\mathrm{AdS}_{p-1}$, for $z^{+} \neq 0$. The time dependence of the solution becomes manifest when one expresses $z^{+}$in terms of the global parametrisation (2) of $\mathrm{AdS}_{p-1}$.

Following the discussion on the conformal boundary for $\mathrm{AdS}_{p}$, it is straightforward to determine the conformal boundary for $\mathrm{AdS}_{p} / \Gamma^{+}$. Using the global parametrization for $\mathrm{AdS}_{p-1}$ appearing in (12), this metric is conformal to

$$
g=g_{E(p-1)}+\frac{1}{2}\left(\cos \tau+\sin \tilde{\varphi} \Omega^{y}\right)^{2}(d z)^{2},
$$

where the non-compact coordinate $\rho$ in $\operatorname{AdS}_{p-1}$ was related to $\tilde{\varphi}$ through $\tan \tilde{\varphi}=\sinh \rho$. Once more, the conformal boundary is at $\tilde{\varphi}=\pi / 2$, since $S^{1}$ does not have one. Notice that $\Omega^{y}$ is just indicating the direction in which the null rotation identification was done. Thus the metric on the conformal boundary is given by

$$
g_{\partial \mathrm{AdS}_{p} / \Gamma^{+}}=-(d \tau)^{2}+g_{S^{p-3}}+\frac{1}{2}\left(\cos \tau+\Omega^{y}\right)^{2}(d z)^{2} .
$$

We would finally like to prove that the conformal boundary of $\mathrm{AdS}_{p} / \Gamma^{+}$ is equivalent to the conformal compactification of $\mathbb{R}^{1, p-2} / \Gamma^{+}$. First of all, we introduce adapted coordinates for $\mathbb{R}^{1,2} \subset \mathbb{R}^{1, p-2}$ where $\Gamma^{+}$acts non-trivially, just as in (9). By standard manipulations for studying the conformal infinity in flat Minkowski spacetime, the metric on $\mathbb{R}^{1, p-2} / \Gamma^{+}$is conformal to

$$
g=-\left(d \tau^{\prime}\right)^{2}+(d \theta)^{2}+(\sin \theta)^{2} g_{S^{p-4}}+\frac{1}{2}\left(\sin \tau^{\prime}+\sin \theta \Omega^{\tilde{y}}\right)^{2}(d z)^{2} .
$$

Of course, the above description also breaks down when $\tilde{y}^{+}=0$. It is though manifest that both (14) and (15) are equivalent if $\Omega^{y}=\sin \theta \Omega^{\tilde{y}}$ 
and $\tau^{\prime}=\tau+\pi / 2$, that is, if the directions of the corresponding null rotations are conveniently identified. Strictly speaking, the above explicit computation shows that the conformal boundary for $\mathrm{AdS}_{p} / \Gamma^{+}$in the adapted coordinate system $\left\{z^{ \pm}, z\right\}$ is equal to the conformal compactification of $\mathbb{R}^{1, p-2} / \Gamma^{+}$in the adapted coordinates $\left\{\tilde{y}^{ \pm}, z\right\}$. On the other hand, even though both sets of coordinates break down in different sets of points, both sets actually coincide when restricting them to the confomal boundary and compactification, respectively. This allows us to state that the conformal boundary of $\operatorname{AdS}_{p} / \Gamma^{+}$ is equal to the conformal compactification of $\mathbb{R}^{1, p-2} / \Gamma^{+}$.

Notice that concentrating on the hypersurface defined by the fixed values $\rho=\rho_{0}, \theta=\theta_{0}$ and zooming the region close to the singularity $\tau_{0}$, by expanding $\tau=\tau_{0}+\delta \tau, \delta \tau \ll 1$, one recovers the double cone scenario advocated in [9]

$g_{A d S_{p} / \Gamma} \sim \frac{R^{2}}{(\cos \tilde{\theta})^{2}}\left\{-(d \delta \tau)^{2}+\left(\sin \tilde{\theta}_{0}\right)^{2}\left(\sin \theta_{0}\right)^{2} g_{S^{p-4}}+\frac{1}{2}\left(\sin \tau_{0}\right)^{2}(\delta \tau)^{2}(d \tilde{y})^{2}\right\}$.

\subsection{BTZ black holes}

The extremal 2+1 BTZ black hole [38] can be understood as the quotient of a subset of $A d S_{3}$ by the discrete action generated by $\xi^{+}$. See [39] for a more detailed discussion. What we would like to address here concerns the embedding of such a construction in higher dimensional $A d S_{p}(p \geq 4)$ spaces. To do so, we set

$$
-u^{2}-v^{2}+x^{2}+y^{2}=-\rho^{2},
$$

and inserting it back into (1), we learn $\rho \in[R, \infty)$. It is thus natural to introduce global coordinates

$$
\begin{aligned}
\rho & =R \cosh \mu \\
x^{i} & =R \sinh \mu \hat{x}^{i}
\end{aligned}
$$

where $\hat{x}^{i}$ parametrise a $(\mathrm{p}-4)$-sphere. Notice that the range of the coordinate $\mu$ introduced in (17), depends on the dimension of the full $A d S_{p}$ space under consideration. In particular,

$$
\begin{aligned}
& p=4 \quad \Rightarrow \quad-\infty<\mu<\infty \\
& p>4 \quad \Rightarrow \quad 0 \leq \mu<\infty .
\end{aligned}
$$


On the other hand, we shall parametrise the $\{u, v, x, y\}$ in terms of Poincaré coordinates, which now will depend on the point $\mu$

$$
y+u=\frac{R \cosh \mu}{z} \quad, \quad x=\frac{R \cosh \mu}{z} \beta \quad v=-\frac{R \cosh \mu}{z} \gamma .
$$

It is precisely when one restricts to a single patch of $A d S_{3}$ with a definite sign of the $\mathrm{z}$ coordinate $(z>0$, for instance), that by modding out the corresponding spacetime by the discrete action generated by $\xi^{+}$one expects to embed the BTZ black hole in $A d S_{p}$. This is explicitly checked by rewriting the induced metric on (11) in the coordinate system defined in (17) and (19). The result is given by

$$
g_{A d S_{p} / \Gamma}=R^{2}\left\{(\cosh \mu)^{2} g_{\mathrm{BTZ}}+(d \mu)^{2}+(\sinh \mu)^{2} g_{S^{p-4}}\right\},
$$

where $g_{\mathrm{BTZ}}$ stands for the $2+1$ massless extremal BTZ black hole metric,

$$
g_{\mathrm{BTZ}}=-r^{2}(d t)^{2}+\frac{(d r)^{2}}{r^{2}}+r^{2}(d \phi)^{2}
$$

which was written in the standard coordinates $t=\gamma, \phi=\beta$ and $r=z^{-1}$, all of them being dimensionless. On the other hand, $g_{S^{p-4}}$ stands for the metric on a unit sphere of dimension $p-4$.

It is worthwhile pointing out that the above embedding of the extremal BTZ black hole in $A d S_{p}(p \geq 4)$, can be straightforwardly generalised to the non-extremal ones. If instead of considering the action generated by $\xi^{+}$, one would have considered the action generated by any of the Killing vectors spanning $\mathrm{SO}(2,2)$ giving rise to non-extremal BTZ black holes (see [39]), then one would have parametrised $\left\{u^{i}\right\}=\{u, v, x, y\}$ by

$$
u^{i}=R \cosh \mu \hat{u}^{i} \quad, \quad-\hat{u}^{2}-\hat{v}^{2}+\hat{x}^{2}+\hat{y}^{2}=-1 .
$$

The choice of $\left\{\hat{u}^{i}\right\}$ is directly related with the choice of discrete quotient (or Killing vector). The discussion on the remaining coordinates $\left\{x^{i}\right\}$ is as before. All in all, one derives the metric involving a non-extremal BTZ black hole $\left(\tilde{g}_{B T Z}\right)$ embedded in $A d S_{p}$ for $p \geq 4$ :

$$
g_{A d S_{p} / \Gamma}=R^{2}\left\{(\cosh \mu)^{2} \tilde{g}_{\mathrm{BTZ}}+(d \mu)^{2}+(\sinh \mu)^{2} g_{S^{p-4}}\right\} .
$$

\section{$3 \quad$ Embedding in String/M-theory}

Even though there are many known examples for embeddings of AdS spaces of different dimensions in string theory, we shall concentrate on the maximally supersymmetric ones. These are of the form $\mathrm{AdS}_{p+2} \times \mathrm{S}^{D-p-2}$ and 


\begin{tabular}{|c|c|c|c|}
\hline Brane & $\mathrm{p}$ & $\mathrm{D}$ & $\iota$ \\
\hline M2 & 2 & 11 & $\frac{1}{2}$ \\
D3 & 3 & 10 & 1 \\
M5 & 5 & 11 & 2 \\
\hline
\end{tabular}

Table 1: Dimensions and radii of curvature

can be thought of near horizon geometries of the M2-, M5- and D3-brane configurations. The values of $p$ and $D$ are listed in Table 1 along with the ratio $\iota=\mathrm{R}_{\mathrm{AdS}_{p+2}} / \mathrm{R}_{\mathrm{S}^{D-p-2}}$ of the radii of curvature of the two factors.

The metric on the direct product $\mathrm{AdS}_{p+2} \times \mathrm{S}^{D-p-2}$ is

$$
g=g_{\mathrm{AdS}_{p+2}}+\left(\mathrm{R}_{\mathrm{S}}\right)^{2} g_{\mathrm{S}^{D-p-2}} .
$$

These configurations do also carry fluxes. Using the same conventions as in 42, these are summarised below :

$$
\begin{array}{ll}
\mathrm{AdS}_{4} \times \mathrm{S}^{7} & : F_{4}=\frac{3}{\mathrm{R}_{\mathrm{AdS}}} \operatorname{dvol}\left(\mathrm{AdS}_{4}\right) \\
\mathrm{AdS}_{5} \times \mathrm{S}^{5} & : F_{5}=\frac{1}{2 \mathrm{R}_{\mathrm{AdS}}}\left(\mathrm{dvol}\left(\mathrm{AdS}_{5}\right)+\operatorname{dvol}\left(\mathrm{S}^{5}\right)\right) \\
\mathrm{AdS}_{7} \times \mathrm{S}^{4} & : F_{4}=\frac{6}{\mathrm{R}_{\mathrm{AdS}}} \operatorname{dvol}\left(\mathrm{S}^{4}\right) .
\end{array}
$$

It is straightforward to extend our previous analysis to these supersymmetric vacua. By construction, the action of $\Gamma^{+}$is restricted to the $\operatorname{AdS}_{p+2}$ part of the direct product, giving rise to $\left(\mathrm{AdS}_{p+2} / \Gamma^{+}\right) \times \mathrm{S}^{D-p-2}$. Therefore, in the local adapted coordinate system (9)

$$
g_{\left(\operatorname{AdS}_{p+2} / \Gamma^{+}\right) \times \mathrm{S}^{D-p-2}}=g_{\mathrm{AdS}_{p+1}}+\left(z^{+}\right)^{2}(d z)^{2}+\left(\mathrm{R}_{\mathrm{S}}\right)^{2} g_{\mathrm{S}^{D-p-2}} .
$$

Concerning the field strengths (23), due to its geometrical content, it is easy to work out their local expressions as

$$
\begin{array}{ll}
\left(\operatorname{AdS}_{4}\right) / \Gamma^{+} \times \mathrm{S}^{7} & : F_{4}=\frac{3}{\mathrm{R}_{\mathrm{AdS}}} z^{+} \operatorname{dvol}\left(\operatorname{AdS}_{3}\right) \wedge d z \\
\left(\mathrm{AdS}_{5}\right) / \Gamma^{+} \times \mathrm{S}^{5} & : F_{5}=\frac{1}{2 \mathrm{R}_{\mathrm{AdS}}} z^{+}\left(\operatorname{dvol}\left(\operatorname{AdS} \mathrm{S}_{4}\right) \wedge d z+\operatorname{dvol}\left(\mathrm{S}^{5}\right)\right)
\end{array}
$$

whereas $F_{4}$ is left unmodified for $\mathrm{AdS}_{7} \times \mathrm{S}^{4}$. Remember that these expressions break down at $z^{+}=0$ and notice that their fluxes over $\mathrm{S}^{D-p-2}$ are unchanged. 
Whenever one considers an orbifold of any supersymmetric vacua, there is an issue concerning the amount of supersymmetry preserved. Just as there is a known correspondence among parallel spinors in $\mathbb{R}^{n+1}$ and Killing spinors in the n-sphere $\left(\mathrm{S}^{n} \hookrightarrow \mathbb{R}^{n+1}\right)$ embedded in it 41 , one can prove that there is an analogous correspondence between parallel spinors in $\mathbb{R}^{2, p-1}$ and Killing spinors on $\mathrm{AdS}_{p} \hookrightarrow \mathbb{R}^{2, p-1}$. In this way, since the null orbifold breaks half of the supersymmetries in $\mathbb{R}^{2, p-1}$, we learn the corresponding orbifolds of the maximally supersymmetric AdS spaces preserve one half of the original supersymmetries. In any case, we give an explicit proof of this fact in the appendix.

\section{Null orbifolds in branes}

When dealing with null orbifolds in flat spacetime, their singularities are naturally smoothed by modifying the action by which one quotients the original manifold. In other words, one considers a different Killing vector from the one given in (5). This gives rise to the null-brane discrete quotient [4, 8] which is associated with the Killing vector

$$
\xi=R \partial_{z}+\xi^{ \pm}
$$

$z$ being some spacelike direction orthogonal to the three dimensional lorentzian space where $\xi^{ \pm}$acts.

As emphasized in [8] and extensively discussed in [32], a sufficient condition for a brane-like configuration to exist in such string vacuum (null-brane) is to have an $\operatorname{ISO}(1,3)$ isometry subgroup. This is the case in which we are going to concentrate, and so it will not apply for M2-branes. The structure of singularities is analogous to the one in flat spacetime, since the branes under consideration are flat. The main difference though, is the existence of horizons associated with brane throats.

We shall concentrate on D3-branes, even though a similar discussion would apply for M5-branes. Following [4], the classical supergravity configuration describing D3-branes in a null-brane vacuum has a ten dimensional metric

$$
\begin{aligned}
g=V^{-1 / 2}\left\{-2 d y^{+}\right. & d y^{-}+d y^{2}+\left(1+\left(\frac{y^{+}}{R}\right)^{2}\right) d z^{2} \\
& \left.+2 d z\left(\frac{y^{+}}{R} d y-\frac{y}{R} d y^{+}\right)\right\}+V^{1 / 2}\left(d r^{2}+r^{2} g_{S^{5}}\right)
\end{aligned}
$$

\footnotetext{
${ }^{4}$ This correspondence was found in collaboration with J.M. Figueroa-O'Farrill and will be discussed in 43$]$.
} 
with constant dilaton and the corresponding self-dual five form flux. The function $V=V(r)$ is the usual harmonic function

$$
V(r)=1+\frac{Q^{4}}{r^{4}} \quad, \quad Q^{4}=2 \pi^{3 / 2} \mathrm{~g}_{\mathrm{YM}}^{2} \mathrm{~N}\left(\alpha^{\prime}\right)^{2} .
$$

Taking the near horizon limit

$$
\alpha^{\prime} \rightarrow 0 \quad, \quad \mathrm{u}=\frac{\mathrm{r}}{\alpha^{\prime}}, \mathrm{g}_{s}, \mathrm{R} \quad \text { fixed }
$$

one is left with the cosmological model

$$
\begin{array}{r}
g=\alpha^{\prime}\left\{\frac { \mathrm { u } ^ { 2 } } { \sqrt { \lambda } } \left(-2 d y^{+} d y^{-}+d y^{2}+\left(1+\left(\frac{y^{+}}{R}\right)^{2}\right) d z^{2}\right.\right. \\
\left.\left.+2 d z\left(\frac{y^{+}}{R} d y-\frac{y}{R} d y^{+}\right)\right)+\frac{\sqrt{\lambda}}{\mathrm{u}^{2}} d \mathrm{u}^{2}+\sqrt{\lambda} g_{S^{5}}\right\}
\end{array}
$$

where $\lambda=2 \pi^{3 / 2} \mathrm{~g}_{\mathrm{YM}}^{2} \mathrm{~N}$ and the corresponding self-dual five form fluxes. The above spacetime just covers the Poincaré patch $(0<u<\infty)$. It is manifest that the effective radius of the compact dimension $z$ vanishes on the horizon $(\mathrm{u}=0)$. At this stage, one may decide to maximally extend the above discrete quotient to global AdS, by crossing the horizon, or whenever such an effective radius becomes smaller than the string scale, one may apply T-duality. This second possibility will be postponed to the last section.

In order to carry on the first possibility, we need to identify how the generator of the discrete quotient in the Poincaré patch $\left(\xi=R \partial_{z}+\xi^{ \pm}\right)$ acts in global AdS. We shall do this for $\mathrm{AdS}_{p+2}$. Let us remind the relation among the coordinates parametrising the Poincaré patch $\left(y^{\mu}, u\right)$ and the ones describing the embedding $\operatorname{AdS}_{p+2} \hookrightarrow \mathbb{R}^{2, p+1}$ (1) :

$$
\begin{aligned}
X^{\mu} & =\frac{u}{R_{\mathrm{AdS}}} x^{\mu} \quad(\mu=0,1, \ldots, p-1) \\
X^{p+1} & =\frac{R_{\mathrm{AdS}}^{2}}{2 u}\left[1+\frac{u^{2}}{R_{\mathrm{AdS}}^{2}}\left(1+\frac{\eta_{\mu \nu} y^{\mu} y^{\nu}}{R_{\mathrm{AdS}}^{2}}\right)\right] \\
X^{p} & =\frac{R_{\mathrm{AdS}}^{2}}{2 u}\left[1-\frac{u^{2}}{R_{\mathrm{AdS}}^{2}}\left(1-\frac{\eta_{\mu \nu} y^{\mu} y^{\nu}}{R_{\mathrm{AdS}}^{2}}\right)\right],
\end{aligned}
$$

where we identified $X^{p+1}=u$ and $X^{0}=v$, without losing generality.

Using the identities :

$$
\frac{\partial X^{\mu}}{\partial y^{\nu}}=\frac{u}{\mathrm{R}_{\mathrm{AdS}}} \delta_{\nu}^{\mu} \quad, \quad \frac{\partial X^{p+1}}{\partial y^{\nu}}=\frac{\partial X^{p}}{\partial y^{\nu}}=\frac{\eta_{\nu \mu} X^{\mu}}{\mathrm{R}_{\mathrm{AdS}}} \quad, \quad X^{p+1}-X^{p}=u
$$


it is manifest that

$$
\begin{gathered}
P_{\mu}=\partial_{\mu} \rightarrow \frac{\sqrt{2}}{\mathrm{R}_{\mathrm{AdS}}}\left(X_{\mu} \partial_{+}-X_{+} \partial_{\mu}\right) \\
L_{\mu \nu}=y_{\mu} \partial_{\nu}+y_{\nu} \partial_{\mu} \rightarrow X_{\mu} \partial_{\nu}+X_{\nu} \partial_{\mu}
\end{gathered}
$$

where $X^{ \pm}=\left(X^{p+1} \pm X^{p}\right) / \sqrt{2}$.

The above computation tells us that Lorentz transformations belonging to $\mathrm{SO}(1, p)$ in the Poincaré patch are mapped into Lorentz transformations in $\mathrm{SO}(2, p+1)$ in global AdS, which in particular justifies our previous analysis on null orbifolds in AdS spaces. Furthermore, translations $\left(\mathrm{R}_{\mathrm{AdS}} \partial_{\mu}\right)$ in the Poincaré patch are mapped to null rotations in $\mathrm{SO}(2, p+1)$ involving "the second time" in $\mathbb{R}^{2, p+1}$. To be more precise,

$$
\mathrm{R}_{\mathrm{AdS}} \partial_{\mu} \rightarrow \sqrt{2} \xi^{-}
$$

as defined in (5). This analysis strongly suggests to analyse the geometrical structure of $\operatorname{AdS}_{p}(p \geq 5)$ modded out by the discrete action generated by $\xi=\xi^{+}(u, x, y)+\xi^{-}(v, s, r)$. This is the subject of the next section.

\section{Null-brane discrete quotients in AdS spaces}

Let us parametrise $\operatorname{AdS}_{p}(p \geq 5)$ as the hyperboloid

$$
-u^{2}+x^{2}+y^{2}-v^{2}+r^{2}+s^{2}+\sum_{i}^{p-5}\left(x^{i}\right)^{2}=-\left(\mathrm{R}_{\mathrm{AdS}}\right)^{2},
$$

and study the quotient manifold $\mathrm{AdS}_{p} / \Gamma^{+-}$obtained by a discrete identification of points using the action $\Gamma^{+-}$associated with the Killing vector

$$
\xi=\xi^{+}(u, x, y)+\xi^{-}(v, s, r)
$$

Introducing coordinates $\left\{x^{ \pm}, x\right\}$ and $\left\{s^{ \pm}, s\right\}$ to describe both $\mathbb{R}^{1,2}$ factors where $\Gamma^{+-}$acts non-trivially and assembling them into a column vector $X$, the generator $g_{0}$ of the orbifold acts as

$$
X=\left(\begin{array}{c}
x^{+} \\
x \\
x^{-} \\
s^{-} \\
s \\
s^{+}
\end{array}\right) \rightarrow g_{o} \cdot X=e^{t \mathcal{J}} X=\left(\begin{array}{c}
x^{+} \\
x+t x^{+} \\
x^{-}+t x+\frac{1}{2} t^{2} x^{+} \\
s^{-} \\
s+t s^{-} \\
s^{+}+t s+\frac{1}{2} t^{2} s^{-}
\end{array}\right)
$$


where the matrix $\mathcal{J}$ is given by

$$
\mathcal{J}=\left(\begin{array}{llllll}
0 & 0 & 0 & 0 & 0 & 0 \\
1 & 0 & 0 & 0 & 0 & 0 \\
0 & 1 & 0 & 0 & 0 & 0 \\
0 & 0 & 0 & 0 & 0 & 0 \\
0 & 0 & 0 & 1 & 0 & 0 \\
0 & 0 & 0 & 0 & 1 & 0
\end{array}\right)
$$

It is clear that such an action has fixed points in the embedding space $\mathbb{R}^{2, p-1}$. These are located at $x^{+}=x=s^{-}=s=0$. The question is whether these points belong to our original manifold, $\mathrm{AdS}_{p}$. It is manifest that they do not satisfy (30) for any finite $\left\{x^{-}, s^{+}\right\}$value, thus allowing us to conclude the absence of fixed points under the action of $\Gamma^{+-}$in $\mathrm{AdS}_{p}$. Analogously, the set of points in $\mathbb{R}^{2, p-1}$ where $\|\xi\|^{2}=0$, that is, $s^{-}=x^{+}=0$, does not belong to $\mathrm{AdS}_{p}$. One thus concludes that the vector field $\xi$ (31) is spacelike everywhere in $\mathrm{AdS}_{p}$. The corresponding quotient manifold $\mathrm{AdS}_{p} / \Gamma^{+-}$must be smooth.

Whenever one constructs quotient manifolds, the non-existence of closed causal curves (CCC's) in it is non-trivial. Notice that the distance between a point and its n'th image in our construction is $|t n| \sqrt{\left(x^{+}\right)^{2}+\left(s^{-}\right)^{2}}$. Using the same argument as before, such distance is always positive in $\mathrm{AdS}_{p}$. Since the integral curves of the Killing vector (31) are geodesic, the above distance is geodesic. Therefore, $\mathrm{AdS}_{p} / \Gamma^{+-}$has no CCC's.

Working in global coordinates (2) allows us to study the action of $\mathrm{\Gamma}^{+-}$ on the conformal boundary of $\mathrm{AdS}_{p}$. It is then easy to check that indeed there are no fixed points for finite $\rho$ (the non-compact spacelike direction in global AdS), but that there is a curve of fixed points on an infinite cylinder of maximal circle base extending along global time $\tau$ in AdS and lying in the ryplane. It is also on this curve that $\|\xi\|^{2}$ vanishes. To understand the origin of these singularities, consider the conditions $x^{+}=s^{-}=0$ in global coordinates plus the fundamental trigonometric identity. This gives the constraint

$$
(\tanh \rho)^{2}\left[\left(\Omega^{r}\right)^{2}+\left(\Omega^{y}\right)^{2}\right]=1,
$$

which can only be satisfied for $\rho \rightarrow \infty$ (conformal boundary) and $\left(\Omega^{r}\right)^{2}+$ $\left(\Omega^{y}\right)^{2}=1$. The latter defines a maximal circle in the ry-plane and enforces $\Omega^{i}=0 \forall \mathrm{i} \neq \mathrm{r}$,s. Describing this circle by $\varphi(0 \leq \varphi<2 \pi)$, the curve on the cylinder is given by

$$
\tau=-\varphi \quad(\bmod \pi)
$$

This discussion allows us to state that if there is any field theory dual description, it would be defined in a singular base space. 
As in the discussion of null orbifolds in previous sections, it is useful to introduce a local adapted coordinate system $\left\{z^{ \pm}, \omega^{ \pm}, z, \phi\right\}$ to describe the geometry of $\mathrm{AdS}_{p} / \Gamma^{+-}$

$$
\begin{aligned}
s^{-}=\omega^{-} & , \quad x^{+}=z^{+} \\
s=\phi \omega^{-} & , \quad x=z+\phi z^{+} \\
s^{+}=\omega^{+}+\frac{1}{2} \omega^{-} \phi^{2} & , \quad x^{-}=z^{-}+\phi z+\frac{1}{2} z^{+} \phi^{2}
\end{aligned}
$$

Notice that (33) is a well-defined coordinate system except at $\omega^{-}=0$, where it manifestly breaks down. When rewriting the embedding equation (30) in terms of $\left\{z^{ \pm}, \omega^{ \pm}, z, \phi\right\}$, it becomes

$$
-2 x^{+} x^{-}-2 \omega^{+} \omega^{-}+z^{2}+\sum_{i=1}^{p-5}\left(x^{i}\right)^{2}=-\left(\mathrm{R}_{\mathrm{AdS}}\right)^{2},
$$

so that it describes $\operatorname{AdS}_{p-1}$ whenever $\omega^{-} \neq 0$. Since $\xi=\partial_{\phi}, \phi$ becomes a compact dimension after the identification. The metric of the corresponding quotient manifold

$$
\begin{aligned}
g_{\mathrm{AdS}_{p} / \Gamma^{+-}} & =g_{\mathrm{AdS}_{p-1}}+\left[\left(\omega^{-}\right)^{2}+\left(z^{+}\right)^{2}\right](d \phi)^{2}+2\left(z^{+}\right)^{2} d \phi d\left(\frac{z}{z^{+}}\right) \\
& =g_{\tilde{\mathcal{M}}}+\|\xi\|^{2}\left(d \phi+A_{1}\right)^{2} .
\end{aligned}
$$

describes an $S^{1}$ fibration over some compact manifold $\tilde{\mathcal{M}}$ with non-trivial gauge field, whose field strength $F_{2}=d A_{1}$ is given by

$$
F_{2}=\frac{2}{\left[\left(\omega^{-}\right)^{2}+\left(z^{+}\right)^{2}\right]^{2}}\left\{\left(\omega^{-}\right)^{2} d z^{+} \wedge d z-\omega^{-} \cdot\left(z^{+}\right)^{2} d \omega^{-} \wedge d\left(\frac{z}{z^{+}}\right)\right\} .
$$

It is easy to identify which is the nature of the conformal boundary in this case. The analysis of symmetries given in the previous section teaches us that the conformal boundary of $\mathrm{AdS}_{p} / \Gamma^{+-}$must be equal to the conformal compactification of $\mathbb{R}^{1, p-2} / \Gamma_{\text {null-brane, }}$ where $\Gamma_{\text {null-brane }}$ stands for the discrete action generated by

$$
\xi_{\text {null-brane }}=R \partial_{z}+\xi^{+}
$$

acting non-trivially on $\mathbb{R}^{1,3}$. This statement can be checked as we did for null orbifolds in $\mathrm{AdS}_{p}$. For concreteness, we shall concentrate on the particular case $p=5$. Take the local description (35) for $\mathrm{AdS}_{5} / \Gamma^{+-}$and parametrise 
$\mathrm{AdS}_{4}$ in global coordinates (2). In particular,

$$
\begin{aligned}
z^{+}=\frac{1}{\sqrt{2}}(u+x) & =\frac{\mathrm{R}_{\mathrm{AdS}}}{\sqrt{2}} \cosh \rho\left(\cos \tau_{\mathrm{AdS}}+\tanh \rho \Omega^{x}\right) \\
\omega^{-}=\frac{1}{\sqrt{2}}(v-\omega) & =\frac{\mathrm{R}_{\mathrm{AdS}}}{\sqrt{2}} \cosh \rho\left(\sin \tau_{\mathrm{AdS}}-\tanh \rho \Omega^{\omega}\right) \\
z & =\mathrm{R}_{\mathrm{AdS}} \sinh \rho \Omega^{z}
\end{aligned}
$$

in such a way that $\Omega^{i}$ parametrise a unit 3-sphere. Extending our previous arguments, it can still be checked that there exists a conformal boundary at $\rho \rightarrow \infty$. The metric on such conformal boundary is

$$
\begin{array}{r}
\tilde{g}=-\left(d \tau_{\mathrm{AdS}}\right)^{2}+g_{S^{2}}+\frac{1}{2}(d \phi)^{2}\left[\left(\sin \tau-\Omega^{\omega}\right)^{2}+\left(\cos \tau_{\mathrm{AdS}}+\Omega^{x}\right)^{2}\right] \\
+2 \sqrt{2} d \phi\left[\left(\cos \tau_{\mathrm{AdS}}+\Omega^{x}\right) d \Omega^{z}-\Omega^{z}\left(d \Omega^{x}-\sin \tau_{\mathrm{AdS}} d \tau_{\mathrm{AdS}}\right)\right] .
\end{array}
$$

On the other hand, the metric on the null-brane vacuum $\mathbb{R}^{1,3} / \Gamma_{\text {null-brane }}$

$$
g=-2 d x^{+} d x^{-}+(d y)^{2}+\left[R^{2}+\left(x^{+}\right)^{2}\right](d \phi)^{2}+2 d \phi\left(x^{+} d y-y d x^{+}\right)
$$

can be rewritten in terms of

$$
\begin{aligned}
x^{ \pm} & =\frac{1}{\sqrt{2}}\left(\tau_{f} \pm r \Omega^{x}\right) \\
y & =r \Omega^{y} .
\end{aligned}
$$

By standard procedures, its conformal compactification is given by

$$
\begin{aligned}
& g=-\left(d \tau_{\mathrm{f}}\right)^{2}+g_{S^{2}}+\frac{1}{2}(d \phi)^{2}\left[2 R^{2}\left(\cos \tau_{\mathrm{f}}+\cos \theta\right)^{2}+\left(\sin \tau_{\mathrm{f}}+\Omega^{x} \sin \theta\right)^{2}\right] \\
&+ 2 \sqrt{2} d \phi\left\{\sin \theta\left(\sin \tau_{\mathrm{f}}+\Omega^{x} \sin \theta\right) d \Omega^{y}\right. \\
&\left.-\Omega^{y}(\sin \theta)^{2} d \Omega^{x}+\Omega^{y}\left(\sin \tau_{\mathrm{f}} \cos \theta d \theta-\cos \tau_{\mathrm{f}} \sin \theta d \tau_{\mathrm{f}}\right)\right\}
\end{aligned}
$$

One thus learns that using the identifications

$$
\begin{aligned}
\tau_{\mathrm{f}} & =\frac{\pi}{2}+\tau_{\mathrm{ads}} \\
\Omega^{z} & =\sin \theta \Omega^{y} \\
\Omega^{x} & =\sin \theta \Omega^{x} \\
\Omega^{\omega} & =\cos \theta
\end{aligned}
$$

both, the conformal compactification (38) and the conformal boundary (36) are equivalent. Notice that the angular identifications in (39) match the 
two directions in which the null rotations are defined in $\mathrm{AdS}_{5}$ with the two directions that the null-brane discrete quotient defines on the conformal compactification of Minkowski spacetime.

When considering the above quotient in string/M-theory, the discussion of the metric is as above. Concerning the field strengths in (23), one only has to deal with the one in type IIB since the $\Gamma^{+-}$action can not be defined in $\mathrm{AdS}_{4}$ and it does not change $F_{4}$ for the $\mathrm{AdS}_{7} \times \mathrm{S}^{4}$ vacuum. Using the local adapted coordinate system for $\left(\mathrm{AdS}_{5} / \Gamma^{+-}\right) \times \mathrm{S}^{5}$

$$
\begin{aligned}
F_{5} & =\frac{1}{2 \mathrm{R}_{\mathrm{AdS}}}\left\{\sqrt{\left(\omega^{-}\right)^{2}+\left(z^{+}\right)^{2}} \operatorname{dvol} \tilde{\mathcal{M}} \wedge d \phi+\operatorname{dvol}\left(\mathrm{S}^{5}\right)\right\} \\
& =\frac{1}{2 \mathrm{R}_{\mathrm{AdS}}}\left\{\|\xi\| \operatorname{dvol} \tilde{\mathcal{M}} \wedge d \phi+\operatorname{dvol}\left(\mathrm{S}^{5}\right)\right\} .
\end{aligned}
$$

Both $\left(\operatorname{AdS}_{5} / \Gamma^{+-}\right) \times \mathrm{S}^{5}$ and $\left(\mathrm{AdS}_{7} / \Gamma^{+-}\right) \times \mathrm{S}^{4}$ preserve one quarter of the spacetime supersymmetry. This is explicitly proved in the appendix, but it is very easy to argue using the embedding $\operatorname{AdS}_{p} \hookrightarrow \mathbb{R}^{2, p-1}$. We have constant Killing spinors $\varepsilon_{0}$ in $\mathbb{R}^{2, p-1}$. The action of $\Gamma^{+-}$preserves those satisfying

$$
\left(\Gamma_{-x}+\Gamma_{\hat{+} s}\right) \varepsilon_{0}=0
$$

where $\hat{+}$ stands for a lightlike direction in the vr-plane, whereas - lies in the uy-plane. Since both $\Gamma_{-x}$ and $\Gamma_{\hat{+} s}$ are nilpotent and commute, the quotient preserves $\nu=1 / 4$ of the original spacetime supersymmetry.

\section{The quest for non-perturbative string cos- mology}

As explained in the introduction, the existence of a compact direction along the worldvolume of the branes, opens up a very natural possibility. Whenever its effective radius becomes smaller than the string scale, one is entitled to apply a T-duality transformation, and move to the T-dual description. Let us concentrate on D3-branes, from now on. We can read such a condition from the corresponding near horizon geometry $(\mathrm{p}=3)$ in (27)

$$
\frac{\mathrm{u}^{2}}{\mathrm{~g}_{\mathrm{YM}}^{2} \mathrm{~N}}\left(R^{2}+\left(x^{+}\right)^{2}\right)<1 .
$$

Before giving further details, let us summarise the different descriptions that we have at our disposal in this particular scenario. We are analysing the open string sector of string theory in a null-brane background. Extending 
the formalism developed in [9] for the parabolic orbifold, and further used for the null-brane in [11, 12], one could in principle study a perturbative analysis on the worldsheet of D3-branes in such vacuum. The expected low energy effective field theory on the branes would be that of $d=1+3$ SYM on the null-brane manifold. This is a gauge theory that can be defined in terms of $\mathcal{N}=4 \mathrm{SYM}$ by requiring the connection $A(X)$ and the scalars transforming in the adjoint representation of $\mathrm{SU}(N)$ to satisfy the boundary conditions

$$
\begin{aligned}
\Phi^{j}\left(g_{o} \cdot X\right) & =\Omega(X) \Phi^{j}(X)[\Omega(X)]^{-1} \quad j=1, \ldots, 6 \\
A\left(g_{o} \cdot X\right) & =\Omega(X) A(X)[\Omega(X)]^{-1}-i d \Omega(X)[\Omega(X)]^{-1}
\end{aligned}
$$

where $\Omega(X)$ stands for an $\mathrm{SU}(N)$ group element describing a gauge transformation, $X$ is a vector parametrising $\mathbb{R}^{1,3}$ and $g_{o}$ is the generator of the null-brane discrete quotient

$$
X=\left(\begin{array}{c}
z \\
x^{+} \\
x \\
x^{-}
\end{array}\right) \quad, \quad g_{o} \cdot X=\left(\begin{array}{c}
z+t R \\
x^{+} \\
x+t x^{+} \\
x^{-}+t x+\frac{1}{2} t^{2} x^{+}
\end{array}\right)
$$

It would be interesting to check whether this gauge theory captures all the low energy effective dynamics of the open string sector at weak coupling.

On the other hand, D-branes are massive charged objects which act as a source for the various supergravity fields. Since these D-branes are excitations over the null-brane vacuum, the corresponding classical D3-brane solution is the one in (26) and mentioned in [32]. Relying on the intuition borrowed from the AdS/CFT correspondence, it seems reasonable to establish some duality relation between the previously defined gauge theory and type IIB in the background (27), even though the string theory dynamics in these backgrounds is still not understood.

It is at this stage that T-duality provides us with a bridge to go beyond the Poincaré patch. Since the gravitational configuration we are dealing with is locally isometric to the standard D3-brane solution, the forementioned classical gravity description becomes reliable when the radius of curvature of $\mathrm{AdS}_{5}$ and $\mathrm{S}^{5}$ are large compared to the string scale

$$
\frac{\mathrm{R}_{\mathrm{AdS}}^{4}}{\left(\alpha^{\prime}\right)^{2}} \sim \mathrm{g}_{\mathrm{YM}}^{2} \mathrm{~N} \gg 1 .
$$

Thus, one would expect T-duality to provide a reliable description whenever

$$
\mathrm{u}^{2}\left(R^{2}+\left(y^{+}\right)^{2}\right)<\left(\mathrm{g}_{\mathrm{YM}}^{2} \mathrm{~N}\right)^{1 / 2} \quad, \quad \mathrm{~g}_{\mathrm{YM}}^{2} \mathrm{~N} \gg 1
$$


This is a time dependent condition, a fact which might not be that surprising since energy in the dual gauge theory is not a conserved quantity. Even though this condition restricts the regime of validity of the T-dual picture, what remains true is that whenever it is reliable, it gives rise to a strongly coupled type IIA configuration

$$
e^{\phi} \sim \frac{g_{s} R}{\sqrt{\alpha^{\prime}}} \frac{\left(\mathrm{g}_{\mathrm{YM}}^{2} \mathrm{~N}\right)^{1 / 4}}{\mathrm{u}\left(R^{2}+\left(y^{+}\right)^{2}\right)^{1 / 2}} \gg 1
$$

which forces us to open up an extra eleven dimension, and look for the corresponding M-theory lift classical configuration. We shall provide such a lift before taking any near horizon limit, just because the corresponding configuration is delocalised in two directions and it would not be that illuminating. This is found to be

$$
\begin{aligned}
g & =V^{1 / 3} \cdot L^{-2 / 3}\left(d x_{\natural}^{2}+d \tilde{z}^{2}\right)+V^{1 / 3} \cdot L^{1 / 3}\left(d r^{2}+r^{2} g_{S^{5}}\right) \\
& +V^{-2 / 3} \cdot L^{1 / 3}\left\{2 d y^{+} d y^{-}+d y^{2}-L^{-1}\left(\frac{y}{R} d y^{+}-\frac{y^{+}}{R} d y\right)^{2}\right\} \\
C_{3} & =V^{-1} d y^{+} \wedge d y^{-} \wedge d y+L^{-1}\left(\frac{y^{+}}{R} d y-\frac{y}{R} d y^{+}\right) \wedge d \tilde{z} \wedge d x_{\natural},
\end{aligned}
$$

where we defined the scalar function

$$
L\left(y^{+}, R\right)=1+\left(\frac{y^{+}}{R}\right)^{2},
$$

$x_{\natural}$ stands for the eleventh dimensional coordinate and all quantities are measured in terms of the eleven dimensional Planck scale. The fact that supergravity does not take into account the physical effect of any winding modes is, as usual, the responsible for the delocalisation of (44). As expected, not only the Lorentz group on the three dimensional M2-branes is broken due to the effect of the original null-brane, but also the transverse Lorentz group is manifestly broken. In particular, the two dimensional subspace spanned by $\left\{x_{\natural}, \tilde{z}\right\}$ shrinks to zero size whenever $y^{+} \gg R$. In the other limit, that is close to the "neck" of spacetime $\left(y^{+} \ll R\right)$, the metric in (44) describes a three dimensional delocalised brane with a pp-wave propagating on it.

It is thus desirable to look for the most general eleven dimensional supergravity configuration compatible with the isometries and supersymmetries of our physical system. Its knowledge would teach us about the effect of the original winding sector in the T-dual picture. This question is currently under investigation. This problem is a very generic one and affects the Tdual descriptions of branes in both, fluxbrane and null-brane sectors in string theory. 
Such an eleven dimensional background would be expected to be dual to some sort of large wave length approximation in the gauge theory. It would certainly be very interesting to study the dynamics of such a gauge theory ๑. Due to its definition, correlation functions should be computable in terms of the known ones in $\mathcal{N}=4 \mathrm{SYM}$ by the method of images. Two point functions with times before and after the neck of spacetime and different spatial wavelengths could teach us about the nature of the neck itself. For instance, it could be that for large wave lengths the penetration probability is exponentially small. That would be interpreted as separation of the two branches of the cosmology.

Finally, we would also like to mention that the source of instability discussed in 12, 14] may also exist in these scenarios, due to the redshift of the energy. If so, it would be interesting to understand its origin on the gauge theory side. Thinking of the black hole as a thermal state, one could look for thermalization effects in the two point function. We hope to come back to some of these issues in the near future.

\section{Acknowledgments}

The author would like to thank O. Aharony, M. Berkooz, N. Drukker, JM. Figueroa-O'Farrill, N. Itzhaki, D. Kutasov, M. Roszali and A. Schwimmer for discussions on the topics presented in this work and for encouragement. It is also a pleasure to thank the University of Edinburgh for hospitality during the intermediate stages of the present work. This research has been supported by a Marie Curie Fellowship of the European Community programme "Improving the Human Research Potential and the Socio-Economic knowledge Base" under the contract number HPMF-CT-2000-00480.

\section{A Explicit supersymmetry analysis}

In this appendix, we determine the amount of supersymmetry preserved by $\operatorname{AdS}_{p} / \Gamma^{+}$and $\operatorname{AdS}_{p} / \Gamma^{+-}$. Since both, $\Gamma^{+}$and $\Gamma^{+-}$just act on $\operatorname{AdS}_{p}$, we shall decompose the ten or eleven dimensional spinors into a convenient tensor product of spinors in AdS and on the sphere. By default, we shall denote tne ones in $\mathrm{AdS}$ by $\varepsilon$. These were determined in [14]. Working in a coordinate system where the AdS metric is described by

$$
g_{\mathrm{AdS}}=(d r)^{2}+e^{2 \mathrm{r}} \eta_{\alpha \beta} d x^{\alpha} d x^{\beta},
$$

\footnotetext{
${ }^{5}$ The following comments were suggested by Moshe Roszali.
} 
it was proved that the Killing spinors $\varepsilon$ were satisfying

$$
\nabla_{\mu} \varepsilon=\frac{1}{2} \Gamma_{\mu} \varepsilon \quad \mu=\alpha, \mathrm{r}
$$

which is solved by

$$
\varepsilon=e^{\mathrm{r} / 2} \varepsilon_{+} \quad ; \quad \varepsilon=\left(e^{-\mathrm{r} / 2}+e^{\mathrm{r} / 2} x^{\alpha} \Gamma_{\underline{\alpha}}\right) \varepsilon_{-} \quad, \quad \Gamma_{\underline{r}} \varepsilon_{ \pm}= \pm \varepsilon_{ \pm} .
$$

The local condition of preservation of supersymmetry under the identification $\Gamma^{+}$and $\Gamma^{+-}$requires the action of $\xi$ on the Killing spinors $\varepsilon$ to vanish 45

$$
\mathcal{L}_{\xi} \varepsilon=\nabla_{\xi} \varepsilon+\frac{1}{4} \nabla_{a} \xi_{b} \Gamma^{a b} \varepsilon=0
$$

Working out both terms, they can be written as :

$$
\begin{aligned}
\nabla_{\xi} \varepsilon & =\frac{1}{2} e^{\mathrm{r}}(\xi \cdot \Gamma) \varepsilon \\
\frac{1}{4} \nabla_{a} \xi_{b} \Gamma^{a b} \varepsilon & =\left(\frac{1}{8}\left(\partial_{\alpha} \bar{\xi}_{\beta}-\partial_{\beta} \bar{\xi}_{\alpha}\right) \Gamma \underline{\alpha} \underline{\beta}-\frac{1}{2} e^{\mathrm{r}}(\xi \cdot \Gamma) \Gamma_{\underline{\underline{r}}}\right) \varepsilon
\end{aligned}
$$

where $\xi \cdot \Gamma=\xi^{\alpha} \Gamma_{\underline{\alpha}}, \xi_{\alpha}=e^{2 \mathrm{r}} \eta_{\alpha \beta} \xi^{\beta}=e^{2 \mathrm{r}} \bar{\xi}_{\alpha}$ and $\left\{\Gamma_{\underline{\mu}}, \Gamma_{\underline{\nu}}\right\}=2 \eta_{\mu \nu}$.

It is manifest that for $\varepsilon=e^{\mathrm{r} / 2} \varepsilon_{+}$condition (48) reduces to the one in flat spacetime

$$
\Gamma_{-\underline{x}} \varepsilon=0
$$

For the second kind of Killing spinors, the one involving $\varepsilon_{-}$, splitting $\alpha=$ $\{\hat{\alpha}, z\}, z$ being the direction in which $\xi$ acts as a translation

$$
\begin{aligned}
\mathcal{L}_{\xi} \varepsilon=\frac{1}{8} e^{-\mathrm{r} / 2} & \left(\partial_{\alpha} \bar{\xi}_{\beta}-\partial_{\beta} \bar{\xi}_{\alpha}\right) \Gamma \underline{\alpha \underline{\beta}} \varepsilon_{-}+e^{\mathrm{r} / 2} R \Gamma_{\underline{z}} \varepsilon_{-} \\
& +e^{\mathrm{r} / 2}\left\{\left(\xi^{\hat{\alpha}} \cdot \Gamma_{\underline{\hat{\alpha}}}\right)+\frac{1}{8}\left(\partial_{\hat{\alpha}} \bar{\xi}_{\hat{\beta}}-\partial_{\hat{\beta}} \bar{\xi}_{\hat{\alpha}}\right) \Gamma \underline{\hat{\alpha} \hat{\beta}} x^{\hat{\gamma}} \cdot \Gamma_{\underline{\hat{\gamma}}}\right\} \varepsilon_{-}=0
\end{aligned}
$$

The first line is independent of $x^{\hat{\gamma}}$ whereas the second line is linear in them. Thus, both must vanish independently. From the first one, we learn

$$
\Gamma_{-\underline{x}} \varepsilon_{-}=\Gamma_{\underline{z}} \varepsilon_{-}=0 \quad \Rightarrow \quad \varepsilon_{-}=0 \quad(R \neq 0)
$$

\footnotetext{
${ }^{6}$ The coordinates in (45) describe AdS in the Poincaré patch and it was proved in (29) that $\xi^{-}$was mapped to $R \partial_{z}$ for some spacelike direction $\mathrm{z}$ transverse to the action generated by $\xi^{+}$.
} 
or

$$
\Gamma_{-\underline{x}} \varepsilon_{-}=0 \quad(R=0)
$$

When $R=0$, it can be shown using gamma matrices identities, the form of $\xi^{+}$and eq. (51) that

$$
\frac{1}{8}\left(\partial_{\hat{\alpha}} \bar{\xi}_{\hat{\beta}}-\partial_{\hat{\beta}} \bar{\xi}_{\hat{\alpha}}\right) \Gamma^{\hat{\alpha} \underline{\beta}} x^{\hat{\gamma}} \cdot \Gamma_{\underline{\hat{\gamma}}} \varepsilon_{-}=-\xi^{\hat{\alpha}} \cdot \Gamma_{\underline{\hat{\alpha}}} \varepsilon_{-}
$$

so that the second line in $(50)$ is automatically satisfied whenever the first is.

We sum up the conclusions of the above computation associating the generator of the orbifold with the amount of supersymmetry $(\nu)$ that is being preserved :

$$
\begin{array}{r}
\xi=\xi^{+}(R=0) \Rightarrow \nu=\frac{1}{2} \\
\xi=\xi^{+}+\xi^{-}(R \neq 0) \Rightarrow \nu=\frac{1}{4} .
\end{array}
$$

\section{References}

[1] G.T. Horowitz and A.R. Steif, Singular string solutions with nonsingular initial data, Phys. Lett. B258, 91 (1991).

[2] A.A. Tseytlin, Exact string solutions and duality, arXiv:hep-th/9407099;

C. Klimcik and A.A. Tseytlin, unpublished (1994); A.A. Tseytlin, unpublished (2001).

[3] J. Khoury, B.A. Ovrut, N. Seiberg, P.J. Steinhardt and N. Turok, From Big Crunch to Big Bang, Phys. Rev. D65 (2002) 086007, arXiv:hep-th/0108187;

N. Seiberg, From Big Crunch to Big Bang : Is it possible?, Talk given at the Francqui Colloquium 2001, arXiv:hep-th/0201039.

[4] J.M. Figueroa-O'Farrill and J. Simón, Generalised supersymmetric fluxbranes, J. High Energy Phys. 12 (2001) 011, arXiv:hep-th/0110170.

[5] V. Balasubramanian, S.F. Hassan, E. Keski-Vakkuri and A. Naqvi, A Space-Time orbifold : A Toy Model for a Cosmological Singularity, arXiv:hep-th/0202187. 
[6] L. Cornalba and M. Costa, A new cosmological scenario in string theory, arXiv:hep-th/0203031.

[7] N. Nekrasov, Milne Universe, Tachyons and Quantum Group, arXiv:hep-th/0203112.

[8] J. Simón, The geometry of null rotation identifications, J. High Energy Phys. 06 (2002) 001, arXiv:hep-th/0203201.

[9] H. Liu, G. Moore and N. Seiberg, Strings in a time-dependent orbifold, J. High Energy Phys. 06 (2002) 045, arXiv:hep-th/0204168.

[10] L. Cornalba, M. Costa and C. Kounnas, A Resolution of the Cosmological Singularity with Orientifolds, Nucl. Phys. B637 (2002) 378-394, arXiv: hep-th/0204261.

[11] H. Liu, G. Moore and N. Seiberg, Strings in time-dependent orbifolds, arXiv:hep-th/0206182.

[12] M. Fabinger and J. McGreevy, On Smooth Time-Dependent Orbifolds and Null Singularities, arXiv:hep-th/0206196.

[13] A. Lawrence, On the instability of $3 d$ null singularities, arXiv:hep-th/0205288.

[14] G.T. Horowitz and J. Polchinski, Instability of Spacelike and Null Orbifold Singularities, arXiv:hep-th/0206228.

[15] S. Elitzur, A. Giveon, D. Kutasov and E. Rabinovici, From big bang to big crunch and beyond, J. High Energy Phys. 06 (2002) 017, arXiv:hep-th/0204189.

[16] B. Craps, D. Kutasov and G. Rajesh, String Propagation in the Presence of Cosmological Singularities, J. High Energy Phys. 06 (2002) 053, arXiv:hep-th/0205101.

[17] O. Aharony, M. Fabinger, G. Horowitz, and E. Silverstein, Clean timedependent string backgrounds from bubble baths, J. High Energy Phys. 07 (2002) 007, arXiv:hep-th/0204158.

[18] D. Birmingham and M. Rinaldi, Bubbles in Anti-de-Sitter Space, arXiv:hep-th/0205246.

[19] V. Balasubramanian and S. Ross, The dual of nothing, arXiv:hep-th/0205290. 
[20] R. Cai, Constant curvature black hole and dual field theory, arXiv:hep-th/0206223.

[21] A. Ghezelbash and R. Mann, Nutty bubbles, arXiv:hep-th/0207123.

[22] A. Buchel, P. Langfelder, and J. Walcher, On time-dependent backgrounds in supergravity and string theory, arXiv:hep-th/0207214.

[23] M. Gutperle and A. Strominger, Spacelike branes, J. High Energy Phys. 04 (2002) 018, arXiv:hep-th/0202210.

[24] C. Chen, D. Gal'tsov, and M. Gutperle, S-brane solutions in supergravity theories, Phys. Rev. D66 (2002) 024043, arXiv:hep-th/0204071.

[25] M. Kruczenski, R. Myers, and A. Peet, Supergravity S-branes, J. High Energy Phys. 05 (2002) 039, arXiv:hep-th/0204144.

[26] N. Deger and A. Kaya, Intersecting S-brane solutions of D=11 supergravity, J. High Energy Phys. 07 (2002) 038, arXiv:hep-th/0206057.

[27] A. Sen, Rolling tachyon, J. High Energy Phys. 04 (2002) 048, arXiv:hep-th/0203211.

[28] A. Sen, Time evolution in open string theory, arXiv:hep-th/0207105.

[29] P. Mukhopadhyay and A. Sen, Decay of Unstable D-branes with Electric Fields, arXiv:hep-th/0208142.

[30] J.M. Maldacena, The Large $N$ limit of superconformal field theories and supergravity, Adv. Theor. Math. Phys. 2 (1998) 231, arXiv:hep-th/9711200;

S.S. Gubser, I.R. Klebanov and A.M. Polyakov, Gauge theory correlators from noncritical string theory, Phys. Lett. B428 (1998) 105, arXiv:hep-th/9802109;

E. Witten, Anti-de Sitter space and holography, Adv. Theor. Path. Phys. 2 (1998) 253, arXiv:hep-th/9802150.

[31] O. Aharony, S.S. Gubser, J.M. Maldacena, H. Ooguri and Y. Oz, Large $N$ field theories, String theory and Gravity, Phys. Rep. 323 (2000) 83386, arXiv: hep-th/9905111.

[32] J.M. Figueroa-O'Farrill and J. Simón, Supersymmetric Kaluza-Klein reductions of M2 and M5-branes, arXiv:hep-th/0208107. 
[33] J.M. Figueroa-O'Farrill and J. Simón, Supersymmetric Kaluza-Klein reductions of $M$-waves and MKK-monopoles, arXiv:hep-th/0208108.

[34] G.T. Horowitz and D. Marolf, A new approach to string cosmology, J. High Energy Phys. 07 (1998) 014, arXiv:hep-th/9805207.

[35] K. Behrndt and D. Lüst, Branes, Waves and AdS Orbifolds, J. High Energy Phys. 07 (1999) 019, arXiv:hep-th/9805207.

[36] B. Ghosh and S. Mukhi, Killing spinors and Supersymmetric AdS orbifolds, J. High Energy Phys. 10 (1999) 021,arXiv:hep-th/9908192.

[37] R. Cai, Constant curvature black hole and dual field theory, arXiv:hep-th/0206223.

[38] M. Bañados, C. Teitelboim and J. Zanelli, Phys. Rev. Lett. 69, 1849 (1992).

[39] M. Bañados, M. Henneaux, C. Teitelboim and J. Zanelli, Geometry of the 2+1 black hole, Phys. Rev. D48, 1506 (1993), arXiv: gr-qc/9302012.

[40] A. Hashimoto and S. Sethi, Holography and String Dynamics in TimeDependent Backgrounds, arXiv:hep-th/0208126.

[41] J.M. Figueroa-O'Farrill, On the supersymmetries on Antide Sitter vacua, Class. Quant. Grav. 16 (1999) 2043-2055, arXiv:hep-th/9902066.

[42] M. Blau, J.M. Figueroa-O'Farrill, C. Hull and G. Papadopoulos, Penrose limits and maximal supersymmetry, Class. Quant. Grav. 19 (2002) L87L95, arXiv:hep-th/0201081.

[43] J.M. Figueroa-O'Farrill and J. Simón, Discrete quotients in AdS spaces, to appear.

[44] H. Lü, C.N. Pope and P.K. Townsend, Domain walls from anti-de Sitter spacetime, Phys. Lett. B391 (1997) 39, arXiv:hep-th/9607164.

[45] J.M. Figueroa-O'Farrill and G. Papadopoulos, Homogeneous fluxes, branes and a maximally supersymmetric solution of M-theory, J. High Energy Phys. 06 (2001), 036, arXiv:hep-th/0105308. 